\title{
Intercostal Space Ossification in a Child with Empyema Thoracis and Rhizomelic Chondrodysplasia Punctata
}

\author{
Tan Jih Huei, ${ }^{* 1,2,3}$ Henry Tan Chor Lip, ${ }^{* 1,2,3}$ Simon Jerome Vendargon ${ }^{4}$ \\ 1 Department of General Surgery, Faculty of Medicine, National University of Malaysia Medical Centre, Kuala Lumpur, Malaysia \\ 2 Clinical Research Centre, Hospital Sultan Ismail, Johor Bahru, Malaysia \\ 3 Department of General Surgery, Hospital Sultanah Aminah, Johor Bahru, Malaysia \\ 4 Department of Cardiothoracic Surgery, Hospital Sultanah Aminah, Johor Bahru, Malaysia
}

\begin{abstract}
Correspondence*: Tan Jih Huei, Department of General Surgery, Hospital Sultanah Aminah, Jalan Abu Bakar, Masjid Sultan Abu Bakar, 80000 Johor Bahru, Johor, Malaysia.

E-mail: huei_87@hotmail.com

(C) 2018, Huei et al,

Submitted: 19-11-2017

Accepted: 25-12-2017

Conflict of Interest: None

Source of Support: Nil

This is an open-access article distributed under the terms of the Creative Commons Attribution License, which permits unrestricted use, distribution, and reproduction in any medium, provided the original work is properly cited.
\end{abstract}

\section{DEAR SIR}

Rhizomelic chondrodysplasia punctata (RCDP) is an autosomal recessive metabolic disorder characterised by symmetric rhizomelic shortening of limbs and skeletal anomalies, dwarfism, microcephaly, micrognathia, cleft palate, ichthyosis, congenital heart disease, seizures, repeated respiratory infections, congenital cataracts, and deafness.[1,2] We report a case of RCDP with empyema thoracis and ossification of intercostal spaces.

A 2-year-old girl (weight $4.8 \mathrm{~kg}$ ) first presented at the age of 4 months with shortening of upper limbs, scissoring of lower limbs, fixed flexion deformity of knee and hip joint contractures. Skeletal survey showed shortening of the humerus and femur, punctate calcification over the shoulders joints, hip and knee joints. There was metaphyseal flaring of the distal humerus, femur and proximal tibia. Coronal clefting was identified over the vertebral bodies. At the age of 1 year, she had lensectomy done for bilateral congenital cataract. During current admission she had fever and tachypnea for 1 day. On examination, she was in severe respiratory distress. Chest radiograph showed a right pleural effusion with collapse consolidation of the right lung. She was ventilated in the paediatric intensive care unit and intravenous ceftriaxone commenced. Initial ultrasound of thorax on day 2 of admission revealed consolidation of the right lower lobe. Blood cultures were positive for streptococcus pneumonia.

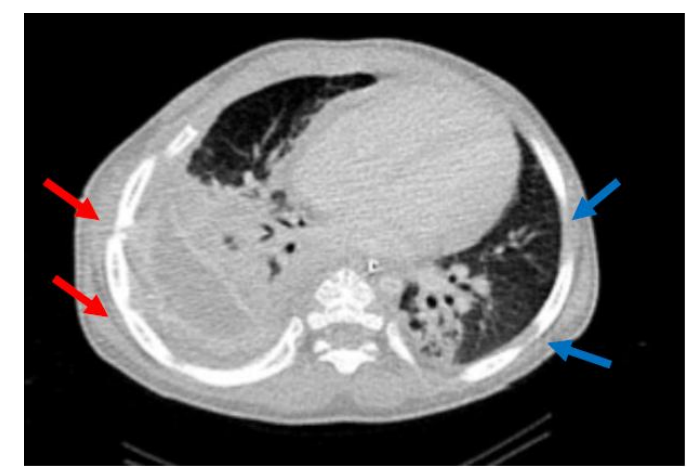

Figure 1: Right intercostal spaces appeared fused with adjacent ribs (red arrow) in compared to left intercostal spaces still having wide space (blue arrow).

A repeated ultrasound on day 4 showed grade 3 empyema thoracis. A right chest tube was inserted and 
drained a total of $350 \mathrm{cc}$ serosanguineous fluid over a period of 5 days. Culture of pleural fluid was negative. Chest tube was removed after 1 week without difficulty. Echocardiography revealed a global pericardial effusion with maximum diameter of $9.2 \mathrm{~mm}$. Computed tomography of thorax revealed a right loculated pleural collection with highest density at lower zone. The surrounding pleura was thickened and enhanced. The right lower lobe was collapsed with consolidation, suggestive of right empyema thoracis (Fig.1). The patient was planned for right thoracotomy and drainage. Due to her stiff neck and limited mouth opening, intubation with laryngoscopy was performed with deep cricoid pressure.

At surgery intercostal spaces were found ossified (Fig.2). The rib spaces were impenetrable either with diathermy or mosquito artery holder. For seeing the risk of excessive bleeding and destruction of right chest wall bony framework, right pleural space was not explored. The child was treated with intravenous ceftriaxone and penicillin for 6 weeks. Chest $x$-ray 10 days following the thoracotomy revealed crowding of the ribs on the right side with ossification of the lateral portion of right intercostal spaces. Child was discharged on clinical improvement three weeks after attempted surgery. Oral penicillin was continued for one month. Chest radiograph on subsequent follow up revealed complete resolution of empyema thoracis.

Handling a child with RCDP for thoracotomy is a challenge to both the anaesthetist and surgeon. The association of cervical spine stenosis, joint stiffness or contracture pose a difficulty in manoeuvrability of the airway.[3] Preoperative anaesthetic evaluation in this patient identified the features of difficult airway such as stiff neck and limited mouth opening. In the index case, additionally we encountered ossified intercostal spaces.

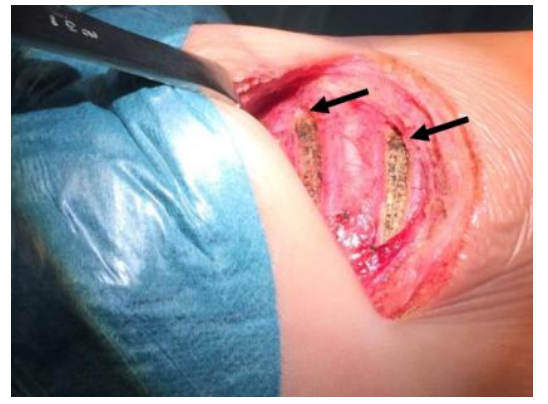

Figure 2: Intraoperative picture of right thoracotomy (2 intercostal spaces shown were charred following failed attempts for entry).

The pleural cavity was inaccessible for evacuation of infective debris and decortication. Interestingly, the chest tube was inserted into right pleural space in the safety triangle 3 weeks prior to the attempted surgery. We are unable to explain this, or this may signify a rapid ossification process of ribs spaces following the pneumonia and right empyema thoracis. Thus, patients with RCDP who present with empyema should warn the treating physicians as to the potential difficulty in gaining entry into the thoracic cavity owing to rapid ossification of intercostal spaces. Medical therapy with longer duration of intravenous antibiotic is an alternative option in such clinical scenario.

\section{REFERENCES}

1. Mahale Y, Kadu VV, Chaudhari A. Rare Case of Rhizomelic Chondrodysplasia Punctata. J Orthop Case Rep. 2015;5:38-40.

2. Huffnagel IC, Clur S-AB, Bams-Mengerink AM, Blom NA, Wanders RJA, Waterham HR, et al. Rhizomelic chondrodysplasia punctata and cardiac pathology. J Med Genet. $2013 ; 50: 419-24$.

3. Bams-Mengerink AM, Majoie CBLM, Duran M, Wanders RJA, Van Hove J, Scheurer CD, et al. MRI of the brain and cervical spinal cord in rhizomelic chondrodysplasia punctata. Neurology. 2006;66(6):798-803. . 